\title{
Elevated risk for obsessive-compulsive symptoms in women pregnant during the COVID-19 pandemic
}

\author{
Brittain L. Mahaffey ${ }^{1} \cdot$ Amanda Levinson $^{1,2} \cdot$ Heidi Preis $^{2,3} \cdot$ Marci Lobel $^{2}$ \\ Received: 24 March 2021 / Accepted: 14 June 2021 / Published online: 16 July 2021 \\ (c) The Author(s), under exclusive licence to Springer-Verlag GmbH Austria, part of Springer Nature 2021, corrected publication 2021
}

\begin{abstract}
The COVID-19 pandemic has led to a public mental health crisis with many people experiencing new or worsening anxiety. Fear of contagion and the lack of predictability/control in daily life increased the risk for problems such as obsessive-compulsive disorder $(\mathrm{OCD})$ in the general population. Pregnant women may be particularly vulnerable to such pandemic-related stressors yet the prevalence of OC symptoms in this population during the pandemic remains unknown. We examined the prevalence of OC symptoms in a sample of 4451 pregnant women in the USA, recruited via targeted online methods at the start of the pandemic. Participants completed self-report measures including the Obsessive-Compulsive Inventory-Revised and the Pandemic-Related Pregnancy Stress Scale. Clinically significant OC symptoms were present in 7.12\% of participants, more than twice as high as rates of peripartum OCD reported prior to the pandemic. Younger maternal age, income loss, and suspected SARS-CoV-2 infection were all associated with higher OC symptoms. Two types of pregnancy-specific stress, pandemic-related and pandemic-unrelated, were both associated with higher levels of OC symptoms. Pandemicrelated pregnancy stress predicted OC symptoms even after controlling for non-pandemic-related, pregnancy-specific stress. Elevated rates of OC symptoms were observed in women pregnant during the pandemic, particularly those experiencing elevated pandemic-related pregnancy stress. This type of stress confers a distinct risk for OC symptoms above and beyond pregnancy-specific stress and demographic factors. Healthcare providers should be prepared to see and treat more peripartum women with $\mathrm{OC}$ symptoms during this and future public health crises.
\end{abstract}

Keywords Pandemic-related pregnancy stress $\cdot$ Pregnancy-specific stress $\cdot$ Obsessive-compulsive symptoms $\cdot$ Perinatal anxiety $\cdot$ COVID-19

The COVID-19 pandemic and consequent public health measures have dramatically altered the lives of millions of people globally, creating a public mental health crisis with high rates of depression, anxiety disorders, and suicidality (Fitzpatrick et al. 2020; Twenge and Joiner 2020a, 2020b). Among the mental health hazards of the pandemic, fear of contagion and the lack of predictability and control in daily life may contribute particularly to vulnerability for

Brittain L. Mahaffey

Brittain.Mahaffey@stonybrookmedicine.edu

1 Department of Psychiatry and Behavioral Health, Renaissance School of Medicine, Stony Brook University, Putnam Hall, New York, NY 11794, USA

2 Department of Psychology, Stony Brook University, New York, NY 11794, USA

3 Department of Pediatrics, Renaissance School of Medicine, Stony Brook University, New York, NY 11794, USA obsessive-compulsive disorder (OCD; Benatti et al. 2020; Davide et al. 2020; Miller and O'Hara 2020).

OCD is characterized by distressing, intrusive thoughts, images, or urges (obsessions) and/or repetitive, ritualistic behaviors intended to neutralize distress (compulsions; APA 2013). Common obsessions include fears of contamination and worries about unintended harm to oneself or others (e.g., unknowingly exposing others to the virus), both of which received substantial media attention during the COVID-19 pandemic. Indeed, rates of OC symptoms in general population samples rose during the pandemic (Abba-Aji et al. 2020; Knowles and Olatunji 2020) and individuals with preexisting OCD reported worsening (Benatti et al. 2020; Chakraborty and Karmakar 2020) or recurrent symptoms (Davide et al. 2020).

Pregnant women may be at particular risk for pandemicrelated OC symptoms (Davenport et al. 2020). Pregnancy is already a time of increased risk for OC symptoms, with 
the prevalence of OCD in pregnancy estimated to be $2-3 \%$ (Fairbrother et al. 2016; Fawcett et al. 2019; Viswasam et al. 2019 ) versus approximately $1 \%$ in the general population (Kessler et al. 2005; Ruscio et al. 2010; Russell et al. 2013). Cognitive-behavioral models link OC symptoms to obsessive beliefs, which include heightened perceived responsibility for preventing harm (Salkovskis 1989), overestimation of threat (Abramowitz et al. 2006; Freeston et al. 1996), and intolerance of uncertainty (Tolin et al. 2003). During pregnancy, obsessive beliefs may take the form of heightened sense of responsibility for the fetus or worries about harm to the pregnancy as a result of contamination/infection (e.g., Buchholz et al. 2020). Furthermore, ritualized checking and avoidance behaviors are part of typical prenatal health routines (e.g., keeping "kick counts," restricting certain foods), thus potentially exacerbating OC symptoms in vulnerable individuals. During the pandemic, threats to health became especially salient for pregnant women worried about contracting SARS-CoV-2 (Corbett et al. 2020). Moreover, unknowns about the health effects of SARS-CoV-2 in pregnancy for both mother and fetus, disruptions of prenatal visits, and alterations to labor and delivery practices fostered increased uncertainty and loss of control-important vulnerability factors for OC symptoms (Gentes \& Ruscio 2011; Gillan et al. 2014).

Given these uncertainties and changes to the experience of pregnancy, it is little wonder that pregnant women experienced high levels of pandemic-related pregnancy stress including feeling unprepared for pregnancy and birth and concerns about infection during the COVID-19 crisis (Preis, et al. 2020b). Irrespective of the pandemic, pregnant women commonly experience concerns about physical symptoms of pregnancy, relationship strains, anxiety about labor and delivery, and concerns about the baby's health and parenting (Lobel et al. 2008). Such pregnancy-specific stress is a robust predictor of adverse birth and health outcomes, even after controlling for maternal health (Ibrahim and Lobel 2020; Lobel et al. 2008). It is also linked to anxiety and mood problems during pregnancy and the postpartum (Ibrahim and Lobel 2020), yet it has rarely been examined as a vulnerability factor for OC symptoms specifically. One goal of the present study, therefore, was to examine whether pregnancy-specific stress contributes to OC symptoms and whether pandemic-related prenatal stress is related to OC symptoms above and beyond other pregnancy-specific stresses.

While elevated rates of prenatal depression, anxiety, and stress have been well-documented since the onset of the COVID-19 pandemic (Hessami et al. 2020; Preis et al. 2020a; Yan et al. 2020), there has been limited examination of prenatal OC symptoms specifically. One report from Turkey suggests very high rates of OCD in pregnant women in hospital settings during the pandemic with upwards of
$61.6 \%$ of pregnant participants vs. $30.7 \%$ of nonpregnant participants reporting clinically significant symptoms (Yassa et al. 2020). Interpretability of these findings, however, is hindered by methodological limitations including that women with a SARS-CoV-2 diagnosis or lifetime history of any psychiatric diagnosis were excluded, and the reasons for hospital presentation were unclear. As such, further study is critically needed to understand the prevalence, symptomatology, and correlates of perinatal OC symptoms during the pandemic.

The current study examined prevalence and severity of OC symptoms in a large sample of pregnant women in the USA at the start of the COVID-19 pandemic, between mid-April and early May, 2020. Based on previous work suggesting unique links between pandemic-related pregnancy stress and maternal mental health (Preis et al. 2020a), we hypothesized that pandemic-related pregnancy stress would predict OC symptoms, over and above pandemic-unrelated pregnancy stress, sociodemographic factors, and obstetric variables.

\section{Methods}

\section{Participants}

During the initial wave of the pandemic, 4,451 pregnant women was recruited between April 24th and May 15th, 2020. Inclusion criteria were being pregnant at the time of recruitment, $\geq 18$ years old, and able to read and write in English. Participants' mean age was $30.84(S D=4.67)$. The sample was predominantly White (92.5\%). Other racial backgrounds reported included 4.7\% Black/African American, 2.8\% Asian American, 2.3\% Native American, and 3.0\% "Other." Hispanic/Latino was also identified with $9.5 \%$.

\section{Study design}

Participants were recruited via advertisements on social media (Facebook, Instagram, and Reddit) for the Stony Brook COVID-19 Pregnancy Experiences (COPE) Study-a longitudinal project to assess psychosocial impacts of the COVID-19 pandemic on pregnant women and their children. Additional details on study recruitment methods are reported elsewhere (Preis et al. 2020a, b, c). The current report utilizes data from the baseline study questionnaire, which included pregnancy-related and general psychological instruments as well as COVID-19-related and obstetric questions, and was administered through Qualtrics, a secure online survey platform. Participants who completed the questionnaire were enrolled in a raffle with a $1 / 100$ chance to win a $\$ 100$ gift card. The study was approved on April 21, 2020 by the Institutional Review Board of Stony Brook University (IRB2020-00227). 


\section{Measures}

\section{Sociodemographic, obstetric, and COVID-19-related factors}

Sociodemographic factors assessed included maternal age (in years), financial status (below average vs. average or above average), relationship status (married or cohabiting vs. single/not cohabiting), and racial identification (White/non-Hispanic vs. person of color). Obstetric factors assessed included parity (nulliparous vs. multiparous), gestational age (weeks pregnant), and high-risk pregnancy status (Yes vs. No or Unsure). COVID-19-related factors assessed included pandemic-related income loss by the participant or someone on whom the participant is dependent (Yes/No), having access to outdoor space (Yes, Whenever I want vs. Sometimes or Rarely), direct contact with a confirmed case of SARS-CoV-2 during pregnancy (Yes/No), and having a suspected but undiagnosed case of SARS-CoV-2 during pregnancy (Yes/No). At the time of data collection, polymerase chain reaction (PCR) testing was not widely available to confirm infection. No participants were excluded due to reporting a diagnosed infection.

\section{OCD screening questions}

All participants were asked the two OCD screening items from the SCID-5 (First et al. 2015), modified to be answered by self-report. The first item assesses obsessions: "Have you ever been bothered by thoughts that didn't make any sense and kept coming back to you even when you tried not to have them?" (Yes/No). The second item assesses compulsions: "Was there ever anything that you had to do over and over again and couldn't resist doing, like washing your hands again and again, counting up to a certain number, or checking something several times to make sure that you'd done it right?" (Yes/No).

\section{Obsessive-Compulsive Inventory-Revised (OCI-R)}

Participants who answered affirmatively to both SCID screening items completed the OCI-R (Foa et al. 2002). While one may meet criteria for a DSM-5 diagnosis of OCD with either obsessions or compulsions, most individuals with clinically significant OC symptoms endorse both obsessions and compulsions (Abramowitz et al. 2014). The OCI-R consists of 18 items rated on a five-point Likert scale $(0=N o t$ at all to $4=$ Extremely) and has been found to have excellent psychometric properties in clinical, nonclinical, and perinatal samples (Abramowitz \& Deacon 2006; Fairbrother et al. 2016; Foa et al. 2002). Previous research indicates that a total OCI-R score (sum of all items, range: $0-72$ ) of $\geq 21$ is a meaningful cutoff for clinically significant OC symptoms (Abramowitz et al. 2005; Belloch et al. 2013; Foa et al. 2002). The OCI-R also yields six subscales (washing, checking, ordering, obsessing, hoarding, and neutralizing). In the current sample, we replicated the 6-factor structure of the OCI-R using CFA (Supplemental Fig. 1) and all subscales achieved acceptable to good internal consistency $(\alpha$ 's $>0.70$, Table 1).

\section{Revised Prenatal Distress Questionnaire (NuPDQ)}

Pregnancy-specific stress was assessed using the NuPDQ (Ibrahim and Lobel 2020; Lobel et al. 2008). Women rate the extent to which they are "feeling bothered, upset, or worried" about 17 pregnancy-relevant stressors (e.g., "physical symptoms of pregnancy"; "what will happen during labor and delivery"; "changes in your weight and body shape") on a scale from $0=$ Not at All to $2=$ Very Much. The NuPDQ score was calculated as the item-level mean (range: 0-2). The NuPDQ achieved good internal consistency in this sample $(\alpha=0.80$; Table 1$)$.

\section{The Pandemic-Related Pregnancy Stress Scale (PREPS)}

COVID-19 pandemic-related prenatal stress was assessed using the PREPS (Preis et al. 2020a, b, c) The PREPS comprises 15 items, rated on a 5-point Likert scale from 1 (Very Little) to 5 (Very Much). In this study, we examined the two PREPS stress subscales, calculated as the mean score of their constituent items (range: 1-5): the preparedness stress scale ( 7 items, e.g., "I am worried that the pandemic could ruin my birth plans") and the perinatal infection stress scale (5 items, e.g., "I am worried that my baby could get COVID19 at the hospital after birth"). The PREPS positive appraisal subscale was not used given lack of theoretical relevance to OC symptoms. The PREPS preparedness and infection stress subscales achieved good internal consistency in this sample ( $\alpha$ 's $\geq 0.80$, see Table 1).

\section{Statistical analyses}

Statistical analyses were performed using SPSS 26.0 (IBM 2017), with the exception of CFAs, which were computed in R (R Core Team, 2018) using the latent variable analysis (lavaan) package (Rosseel 2012). We first examined associations of sociodemographic, obstetric, and COVID-related factors with $\mathrm{OC}$ screening items, using Chi-square tests for dichotomous variables and independent $t$ tests for continuous variables. Similarly, we examined zero-order correlations of OCI-R scores (total and subscales) with sociodemographic, obstetric, and COVID-19-related factors, and with prenatal stress, both related and unrelated to the pandemic (PREPS 


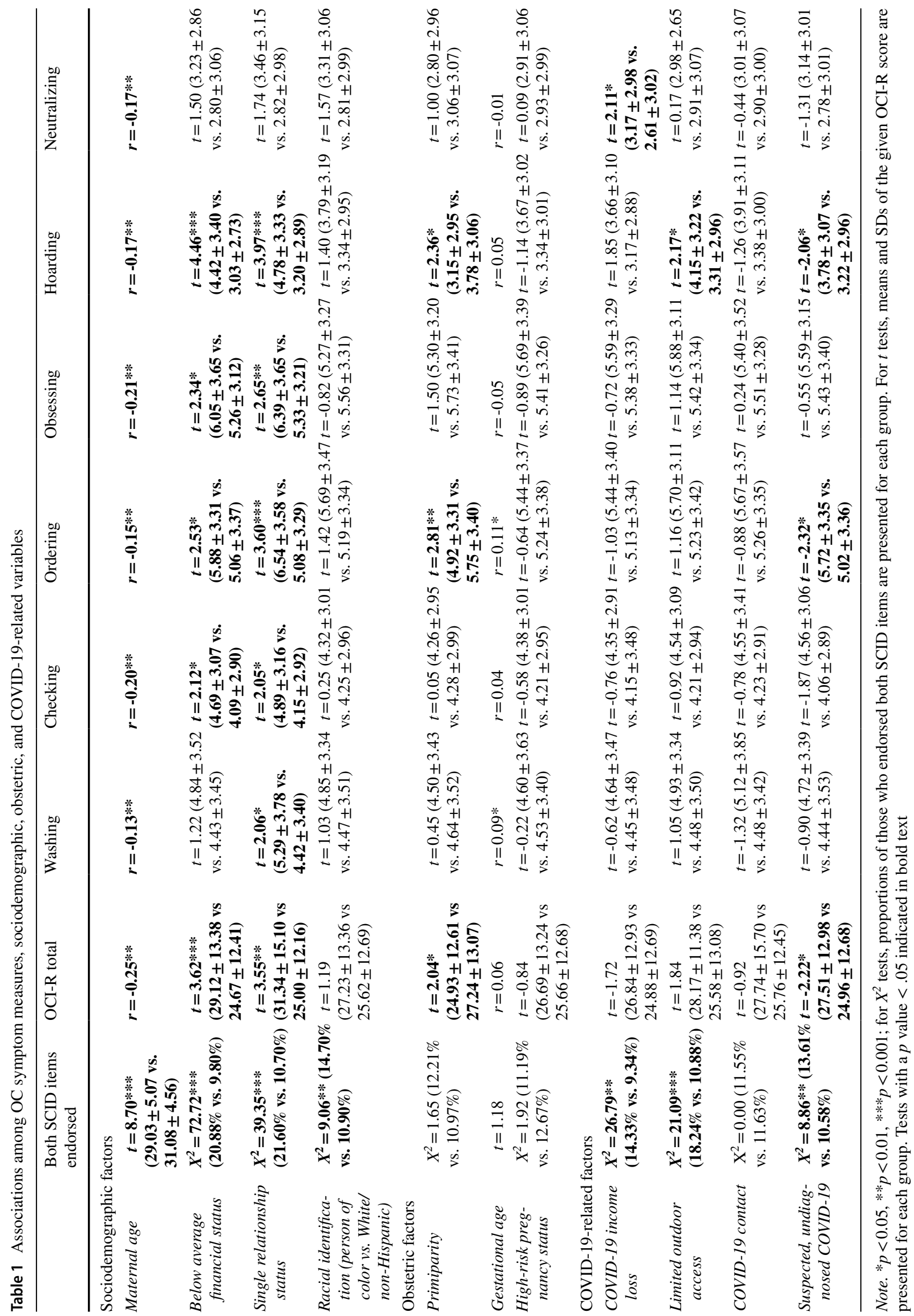


and NuPDQ, respectively). Finally, we constructed hierarchical linear regression models for each of the OCI-R subscales and OCI-R total score, whereby we regressed each OCI-R-dependent variable on PREPS preparedness and infection subscales after controlling for NuPDQ score and for sociodemographic, obstetric, and COVID-19-related factors which were significantly correlated with that OCI-Rdependent variable. Test statistics with $p$ values $<0.05$ are reported as significant.

\section{Results}

\section{Sociodemographic, obstetric, and COVID-19-related factors and OC symptoms}

Table 1 presents associations of sociodemographic, obstetric, and COVID-19-related factors with SCID OCD screening items and OCI-R symptom scales and total OCI-R. Overall, $40.00 \%$ of participants endorsed the SCID obsessions item and $15.26 \%$ endorsed the SCID compulsions item. Thirty-two percent endorsed only 1 item, and $11.59 \%$ endorsed both items. Of the participants endorsing both, $7.12 \%(n=315)$ reached the threshold for clinical significance (OCI-R $\geq 21$ ).

As presented in Table 1, those who endorsed both OCD screening items, and therefore completed the OCI-R, were younger; more likely to report below average financial status, single/not cohabitating relationship status, COVID-19-related income loss, and limited access to outdoor spaces; and more likely to have had a suspected case of SARS-CoV-2 than those who did not endorse either screening item. African American/Black identity, parity, gestational age, highrisk pregnancy, and contact with a confirmed SARS-CoV-2 case did not predict endorsement of OC screening items. Likewise, higher OCI-R total and subscale scores were also associated with younger maternal age, below average financial status, single/not cohabitating relationship status, nulliparity, later gestational age at assessment, COVID19-related income loss, limited outdoor access, and having a suspected case of SARS-CoV-2 (see Table 1).

Table 2 presents correlations among the OCI-R, NuPDQ, and PREPS. Total OCI-R and all OCI-R subscales demonstrated small to medium correlations with the NuPDQ and with the PREPS preparedness and infection subscales ( $r$ 's ranged from 0.10 to 0.36 ).

\section{Hierarchical regression models predicting OCD symptoms}

Table 3 displays all hierarchical regression models, one model predicting each OCI-R score (total and subscales). in each model included all sociodemographic, obstetric, or COVID-19-related factors that exhibited a significant bivariate association with the dependent variable for the given model. Pandemic-related income loss and outdoor access were dropped from these analyses as they did not predict any relevant dependent variables.

for each model included the mean NuPDQ score. Higher NuPDQ scores significantly predicted OCI-R total and all OCI-R subscales ( $\beta$ 's ranged from 0.12 to 0.30 ).

included PREPS preparedness and infection subscales. Total OCI-R score was significantly predicted by PREPS infection $(\beta=0.17)$. Among the subscales, OCI-R washing was significantly predicted by both PREPS subscales (preparedness, $\beta=0.14$, and infection, $\beta=0.31$ ). OCI-R checking symptoms were significantly predicted by PREPS preparedness $(\beta=0.16)$. OCI-R obsessing and neutralizing symptoms were significantly predicted by PREPS infection ( $\beta$ 's $=0.17$, and 0.13 , respectively). Neither PREPS score predicted ordering or hoarding symptoms.

\section{Discussion}

The present study examined the prevalence and correlates of OC symptoms in pregnant women during the early months of the COVID-19 pandemic (April to May, 2020). As hypothesized, rates of clinically significant OC symptoms were more than twice that generally observed in pregnant women prior to the pandemic (7.12\% vs. 2-3\%; Fairbrother et al. 2016; Fawcett et al. 2019; Viswasam et al. 2019). Nonetheless, this rate is substantially lower than the nearly $62 \%$ rate reported by Yassa and colleagues (2020) from a study with several methodological limitations. The rate of OC symptoms observed in the present study is more in line with what one might expect based on pre-pandemic prevalence in perinatal samples. These findings are also consistent with a recent report which found that OC symptoms, particularly those related to contamination, were elevated among women in the postpartum during the COVID-19 pandemic (Thompson and Bardone-Cone 2021) and general population samples (e.g., Samuels et al. 2021).

Sociodemographic variables that predicted greater endorsement of both SCID screening items included younger maternal age, lower financial status, and single/not cohabitating relationship status. COVID-19-related factors that predicted greater OC symptoms included pandemic-related income loss, limited outdoor access, and having a suspected but undiagnosed case of SARS-CoV-2. Among participants who completed the OCI-R, the same socioeconomic and 


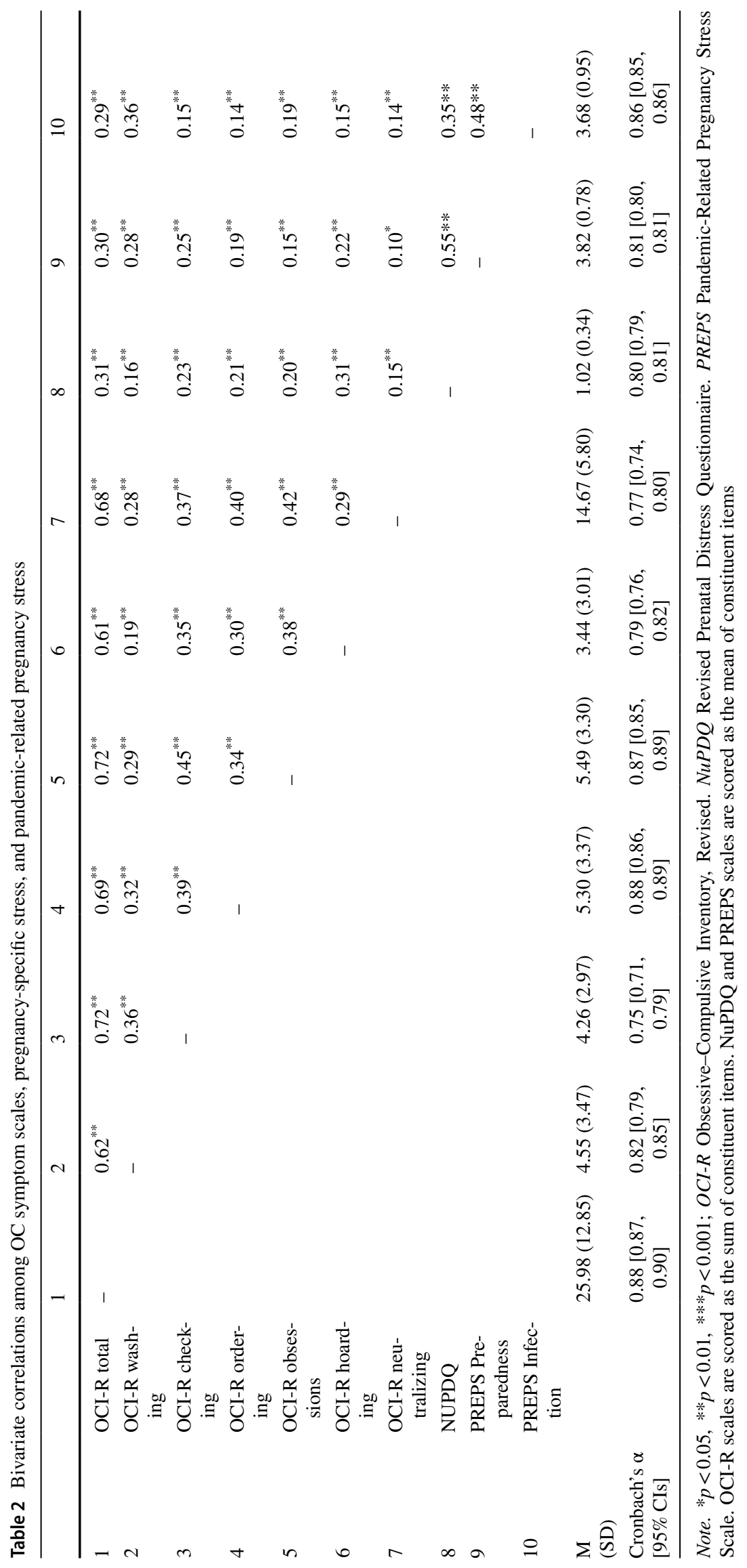




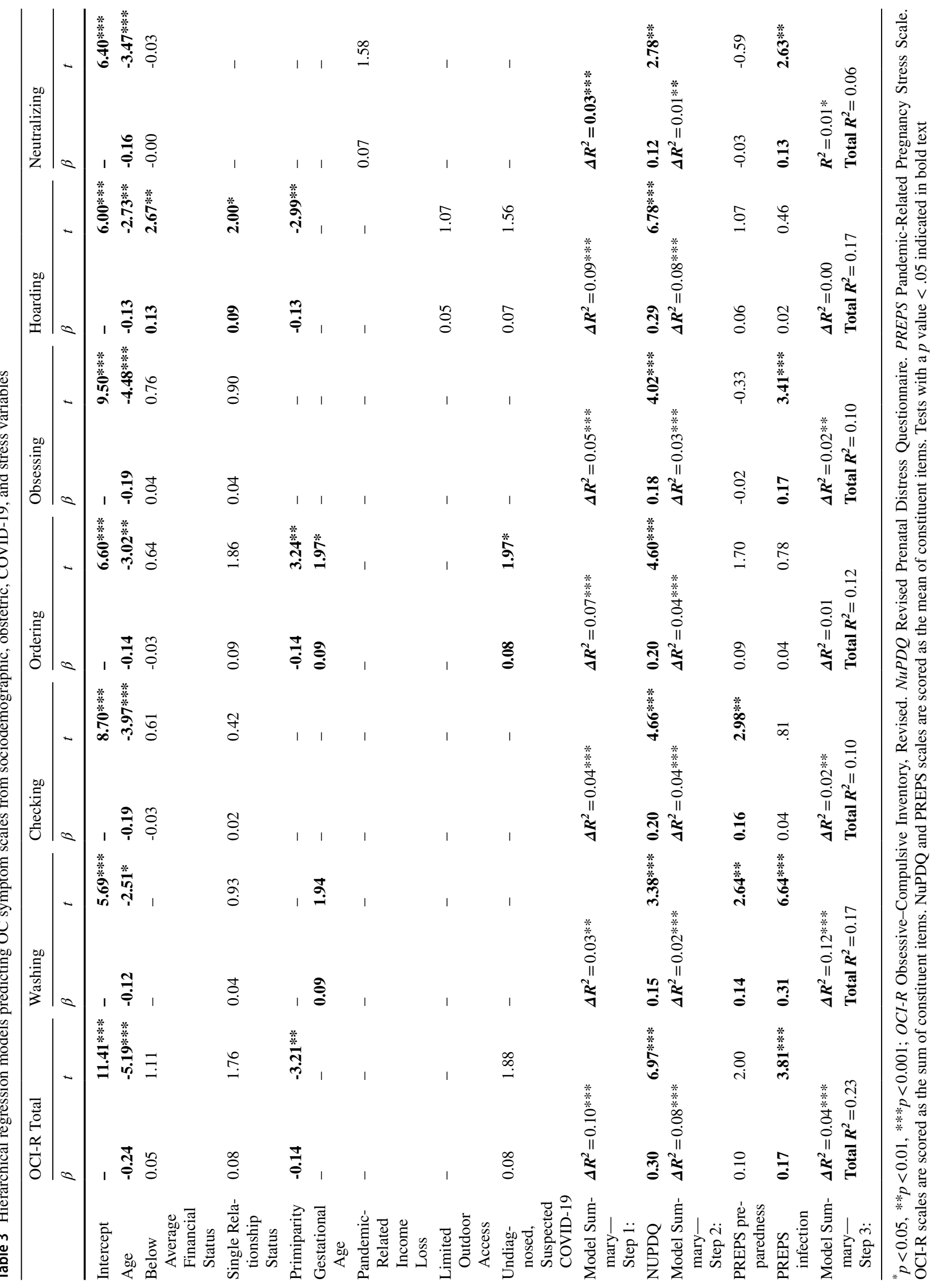


COVID-19-related factors predicted elevated levels of OC symptoms. This speaks to the disproportionate impact of the pandemic on the mental health of socioeconomically disadvantaged women and those personally affected by the pandemic.

Among obstetric factors, greater gestational age at assessment was associated with elevated washing and ordering symptoms. This may reflect increasing urges to prepare for the arrival of a baby towards the end of pregnancy, as well as increasing fears of infection nearing delivery. Nulliparity was correlated with greater OCI-R total scores as well as elevations in hoarding and ordering symptoms. This finding is consistent with previous work suggesting higher rates of perinatal OCD among first-time mothers (Uguz et al. 2007).

As hypothesized, OC symptoms were also significantly associated with greater levels of prenatal stress, both related and unrelated to the pandemic. Furthermore, pandemicrelated stress uniquely contributed to the prediction of elevated OC symptoms including washing, checking, obsessing, and neutralizing symptoms, even after controlling for pandemic-unrelated pregnancy stress. This suggests that the specific types of strain pregnant women experienced due to the pandemic (e.g., fears of contagion and impact of infection on self and fetus, scarcity of resources, disruptions to prenatal care) may confer a distinct influence on risk for OC symptoms.

Of note, different types of pandemic-related stress were also uniquely associated with different OC symptom domains. Specifically, both PREPS subscales (i.e., infection stress and preparedness stress) predicted increased washing, likely reflecting the salience of contamination concerns during the early months of the pandemic. Similarly, obsessing and neutralizing were predicted by infection stress, again likely reflecting the predominance of infection-related worries and associated urges to neutralize. Checking symptoms, in contrast, were predicted only by preparedness stress, suggesting that checking behaviors may have been linked to preparing for childbirth in whatever ways were attainable during the pandemic.

\section{Limitations}

The present study is among the first to examine OC symptoms in a large cohort of US women pregnant during the first wave of the COVID-19 pandemic. A range of psychosocial factors and mental health variables were examined, allowing multivariate analyses and identification of predictors of risk for OC symptoms. Participants in the present study, however, were self-selected and as such, our data do not establish true population prevalence. Moreover, while women identifying with a number of different racial and ethnic backgrounds participated, women of color were underrepresented. Participants were only recruited from the USA, precluding international comparisons. Finally, OC symptoms and suspected SARS-CoV-2 infection were assessed via selfreport. Future work would be strengthened by multimode, multimethod assessment including clinician-administered psychodiagnostic interviews and the recruitment of representative samples including women with medically verified SARS-CoV-2 infections as well women recruited from clini$\mathrm{cal} /$ hospital settings. Finally, longitudinal work is needed to examine possible mediators and moderators of OC symptom risk and to determine whether elevated symptoms persist across waves of the pandemic and into the postpartum.

\section{Clinical and public health implications}

Elevation of OC symptoms in pregnant women during the COVID-19 pandemic carries serious public health implications. OCD during pregnancy is associated with adverse obstetric outcomes including lower birth weight and younger gestational age at birth (Uguz et al. 2015). Perinatal anxiety disorders, such as OCD, are also associated with reduced parental self-efficacy, poorer maternal quality of life (Challacombe et al. 2016; Gezginç et al. 2008), and infant attachment problems (Challacombe et al. 2016; Miller \& O'Hara 2020). Moreover, without treatment, perinatal OC symptoms may become chronic (House et al. 2016). As such, increased prenatal OC symptoms during the pandemic may contribute to persistent maternal mental health problems and adversity for children born during the pandemic. Obstetricians, midwives, and other healthcare providers should be trained to recognize and talk with their patients about the signs and symptoms of perinatal OCD.

In sum, these findings suggest that the unique stressors experienced by pregnant women during the COVID-19 pandemic confer a distinct risk for mental health problems, particularly OC symptoms. Given the severity of the consequences of prenatal maternal OC symptoms for maternal and infant health, parenting, and longer term developmental outcomes, it is imperative that we work to better identify, alleviate, and prevent the development of perinatal OC symptoms during this and future public health crises.

\section{Author' contributions}

Brittain Mahaffey, Marci Lobel, and Heidi Preis were responsible for study conception and design, as well as material preparation and data collection. Data analysis were performed by Amanda Levinson. The first draft of the manuscript was written by Brittain Mahaffey and Amanda Levinson and all authors commented on all versions of the manuscript. All authors read and approved the final manuscript. 
Supplementary Information The online version contains supplementary material available at https://doi.org/10.1007/s00737-021-01157-w.

Acknowledgements We would like to thank all the study participants for their time and their willingness to share their experiences from being pregnant during the COVID-19 pandemic.

We would also like to thank the research assistants who helped in carrying out this project: Francine Chirico, Ilka St. Denis, Lucero Molina, Katya Potkin, Emily Rehbein, Daniella Taboada, and Donna Wilcox.

Funding Funding for this study was provided by a Stony Brook University Office of the Vice President for Research and Institute for Engineering-Driven Medicine COVID-19 Seed Grant. Brittain Mahaffey received support from NIH grant K23HD092888 during preparation of this article. Heidi Preis received support from NIH grant R21DA049827 during preparation of this manuscript.

\section{Declarations}

Ethics approval The current study was approved by the Institutional Review Board of Stony Brook University, in compliance with the standards of the 1964 Helsinki Declaration.

Consent to participate Informed consent was obtained from all individual participants included in the study.

Consent for publication The current manuscript does not present any individual, identifiable information. Patients signed informed consent regarding publishing their data.

Conflicts of interest The authors declare no competing interests.

\section{References}

Abba-Aji A, Li D, Hrabok M, Shalaby R, Gusnowski A, Vuong W, Surood S, Nkire N, Li X-M, Greenshaw AJ (2020) COVID-19 Pandemic and mental health: prevalence and correlates of newonset obsessive-compulsive symptoms in a Canadian province. Int J Environ Res Public Health 17(19):6986

Abramowitz JS, Deacon BJ (2006) Psychometric properties and construct validity of the Obsessive-Compulsive Inventory-Revised: Replication and extension with a clinical sample. J Anxiety Disord 20(8):1016-1035

Abramowitz JS, Fabricant LE, Taylor S, Deacon BJ, McKay D, Storch EA (2014) The relevance of analogue studies for understanding obsessions and compulsions. Clin Psychol Rev 34(3):206-217

Abramowitz JS, Khandker M, Nelson CA, Deacon BJ, Rygwall R (2006) The role of cognitive factors in the pathogenesis of obsessive-compulsive symptoms: A prospective study. Behav Res Ther 44(9):1361-1374

Abramowitz JS, Tolin DF, Diefenbach GJ (2005) Measuring change in OCD: sensitivity of the Obsessive-Compulsive Inventory-Revised. J Psychopathol Behav Assess 27(4):317-324

American Psychiatric Association (2013) Diagnostic and statistical manual of mental disorders, 5th edn. Author, Arlington

Belloch A, Roncero M, García-Soriano G, Carrió C, Cabedo E, Fernández-Álvarez H (2013) The Spanish version of the Obsessive-Compulsive Inventory-Revised (OCI-R): Reliability, validity, diagnostic accuracy, and sensitivity to treatment effects in clinical samples. J Obsess-Compuls Rel 2(3):249-256
Benatti B, Albert U, Maina G, Fiorillo A, Celebre L, Girone N, Fineberg N, Bramante S, Rigardetto S, Dell'Osso B (2020) What happened to patients with Obsessive Compulsive Disorder during the COVID-19 pandemic? A multicentre report from tertiary clinics in northern italy. Front Psych 11:720

Buchholz JL, Hellberg SN, Abramowitz JS (2020) Phenomenology of perinatal obsessive-compulsive disorder. In Biomarkers of Postpartum Psychiatric Disorders (pp. 79-93). Academic Press

Chakraborty A, Karmakar S (2020) Impact of COVID-19 on Obsessive Compulsive Disorder (OCD). Iran J Psychiatry 15(3):256

Challacombe FL, Salkovskis PM, Woolgar M, Wilkinson EL, Read J, Acheson R (2016) Parenting and mother-infant interactions in the context of maternal postpartum obsessive-compulsive disorder: Effects of obsessional symptoms and mood. Infant Behav Dev 44:11-20

Corbett GA, Milne SJ, Hehir MP, Lindow SW, O'connell MP (2020) Health anxiety and behavioural changes of pregnant women during the COVID-19 pandemic. Eur J Obstet Gynecol Reprod Biol 249:96

Davenport MH, Meyer S, Meah VL, Strynadka MC, Khurana R (2020) Moms are not OK: COVID-19 and maternal mental health. Frontiers in Global Women's Health 1:1

Davide P, Andrea P, Martina O, Andrea E, Davide D, Mario A (2020) The impact of the COVID-19 pandemic on patients with OCD: effects of contamination symptoms and remission state before the quarantine in a preliminary naturalistic study. Psychiatry Res 291:113213

Fairbrother N, Janssen P, Antony MM, Tucker E, Young AH (2016) Perinatal anxiety disorder prevalence and incidence. J Affect Disord 200:148-155

Fawcett EJ, Fairbrother N, Cox ML, White IR, Fawcett JM (2019) The prevalence of anxiety disorders during pregnancy and the postpartum period: a multivariate Bayesian meta-analysis. J Clin Psychiatry 80(4):0-0

First M, Williams J, Karg R, Spitzer R (2015) Structured clinical interview for DSM-5-Research version (SCID-5 for DSM-5, research version; SCID-5-RV). American Psychiatric Association, Arlington

Fitzpatrick KM, Harris C, Drawve G (2020) How bad is it? Suicidality in the middle of the COVID-19 pandemic. Suicide and LifeThreatening Behavior 50(6):1241-1249

Foa EB, Huppert JD, Leiberg S, Langner R, Kichic R, Hajcak G, Salkovskis PM (2002) The Obsessive-Compulsive Inventory: development and validation of a short version. Psychol Assess 14(4):485

Freeston MH, Rhéaume J, Ladouceur R (1996) Correcting faulty appraisals of obsessional thoughts. Behav Res Ther 34(5-6):433-446

Gentes EL, Ruscio AM (2011) A meta-analysis of the relation of intolerance of uncertainty to symptoms of generalized anxiety disorder, major depressive disorder, and obsessive-compulsive disorder. Clin Psychol Rev 31(6):923-933

Gezginç K, Uguz F, Karatayli S, Zeytinci E, Aşkin R, Güler Ö, Şahin F, Murat Emül H, Özbulut Ö, Geçici Ö (2008) The impact of obsessive-compulsive disorder in pregnancy on quality of life. Int J Psychiatry Clin Pract 12(2):134-137

Gillan CM, Morein-Zamir S, Durieux A, Fineberg NA, Sahakian BJ, Robbins TW (2014) Obsessive-compulsive disorder patients have a reduced sense of control on the illusion of control task. Front Psychol 5:204

Hessami K, Romanelli C, Chiurazzi M, Cozzolino M (2020) COVID19 pandemic and maternal mental health: a systematic review and meta-analysis. J Matern Fetal Neonatal Med 1-8. https://doi.org/ 10.1080/14767058.2020.1843155

House SJ, Tripathi SP, Knight BT, Morris N, Newport DJ, Stowe ZN (2016) Obsessive-compulsive disorder in pregnancy and the 
postpartum period: course of illness and obstetrical outcome. Arch Womens Ment Health 19(1):3-10

IBM Corp. Released (2017) IBM SPSS Statistics for Windows, Version 25.0. Armonk, NY: IBM Corp.

Ibrahim SM, Lobel M (2020) Conceptualization, measurement, and effects of pregnancy-specific stress: Review of research using the original and revised Prenatal Distress Questionnaire. J Behav Med 43(1):16-33

Kessler RC, Chiu WT, Demler O, Walters EE (2005) Prevalence, severity, and comorbidity of 12-month DSM-IV disorders in the National Comorbidity Survey Replication. Arch Gen Psychiatry 62(6):617-627

Knowles KA, Olatunji BO (2020) Anxiety and safety behavior usage during the COVID-19 pandemic: The prospective role of contamination fear. J Anxiety Disord 77:102323

Lobel M, Cannella DL, Graham JE, DeVincent C, Schneider J, Meyer BA (2008) Pregnancy-specific stress, prenatal health behaviors, and birth outcomes. Health Psychol 27(5):604

Miller ML, O'Hara MW (2020) Obsessive-compulsive symptoms, intrusive thoughts and depressive symptoms: a longitudinal study examining relation to maternal responsiveness. J Reprod Infant Psychol 38(3):226-242

Preis H, Mahaffey B, Heiselman C, Lobel M (2020a) Pandemic-related pregnancy stress and anxiety among women pregnant during the coronavirus disease 2019 pandemic. Am J Obstet Gynecol MFM 2(3): 100155

Preis H, Mahaffey B, Heiselman C, Lobel M (2020b) Vulnerability and resilience to pandemic-related stress among US women pregnant at the start of the COVID-19 pandemic. Soc Sci Med 266:113348

Preis H, Mahaffey B, Lobel M (2020c) Psychometric properties of the pandemic-related pregnancy stress scale (PREPS). J Psychosom Obstet Gynecol 41(3):191-197

R Core Team (2018) R: A language and environment for statistical computing. R Foundation for Statistical Computing, Vienna, Austria. http://www.R-project.org/

[Record \#577 is using a reference type undefined in this output style.]

Rosseel Y (2012) Lavaan: An R package for structural equation modeling and more. J Stat Softw 48(2):1-36 (Version 0.5-12 (BETA))

Ruscio AM, Stein DJ, Chiu WT, Kessler RC (2010) The epidemiology of obsessive-compulsive disorder in the National Comorbidity Survey Replication. Mol Psychiatry 15(1):53-63

Russell EJ, Fawcett JM, Mazmanian D (2013) Risk of obsessivecompulsive disorder in pregnant and postpartum women: a metaanalysis. J Clin Psychiatry 74(4):377-385
Salkovskis PM (1989) Cognitive-behavioural factors and the persistence of intrusive thoughts in obsessional problems. Behav Res Ther 27(6):677-682

Samuels J, Holingue C, Nestadt PS, Bienvenu OJ, Phan P, Nestadt G (2021) Contamination-related behaviors, obsessions, and compulsions during the COVID-19 pandemic in a United States population sample. Journal of Psychiatric Research 138:155-162

Thompson KA, Bardone-Cone A M (2021) 2019-nCOV distress and depressive, anxiety and OCD type, and eating disorder symptoms among postpartum and control women. Arch Womens Ment Health. 1-10. https://doi.org/10.1007/s00737-021-01120-9

Tolin DF, Abramowitz JS, Brigidi BD, Foa EB (2003) Intolerance of uncertainty in obsessive-compulsive disorder. J Anxiety Disord 17(2):233-242

Twenge JM, Joiner TE (2020a) Mental distress among US adults during the COVID-19 pandemic. J of Clin Psycholo 76(12):2170-2182

Twenge JM, Joiner TE (2020b) US Census Bureau-assessed prevalence of anxiety and depressive symptoms in 2019 and during the 2020 COVID-19 pandemic. Depress Anxiety 37(10):954-956

Uguz F, Gezginc K, Zeytinci IE, Karatayli S, Askin R, Guler O, Sahin FK, Emul HM, Ozbulut O, Gecici O (2007) Obsessive-compulsive disorder in pregnant women during the third trimester of pregnancy. Compr Psychiatry 48(5):441-445

Uguz F, Yuksel G, Karsidag C, Guncu H, Konak M (2015) Birth weight and gestational age in newborns exposed to maternal obsessivecompulsive disorder. Psychiatry Res 226(1):396-398

Viswasam K, Eslick GD, Starcevic V (2019) Prevalence, onset and course of anxiety disorders during pregnancy: A systematic review and meta analysis. J Affect Disord 255:27-40

Yan H, Ding Y, Guo W (2020) Mental Health of Pregnant and Postpartum Women During the Coronavirus Disease 2019 Pandemic: A Systematic Review and Meta-Analysis. Front Psychol 11:3324

Yassa M, Yassa A, Yirmibeş C, Birol P, Ünlü UG, Tekin AB, Sandal K, Mutlu MA, Çavuşoğlu G, Tug N (2020) Anxiety levels and obsessive compulsion symptoms of pregnant women during the COVID-19 pandemic. Turk J Obstet Gynecol 17(3):155

Publisher's note Springer Nature remains neutral with regard to jurisdictional claims in published maps and institutional affiliations. 